\title{
Editorial
}

\section{Raisons de croire et raisons d'espérer}

\author{
O. Jeanneret ${ }^{1}$
}

Dans l'arsenal des ressources dont dispose la prévention, l'éducation pour la santé semble l'objet, sous nos latitudes, d'un regain d'intẻrêt de la part des personnels de santé et d'un début d'intérêt du côté des cliniciens.

Quelles explications donner à ce phénomène?

Parmi toutes celles qui se présentent à l'esprit et à l'examen de la littérature récente, trois au moins méritent de retenir notre attention.

En premier lieu, on commence, un peu partout, à se rendre compte que les examens médicaux préventifs - dépistages et surtout bilans de santé - coûtent fort cher pour un rendement et une rentabilité problématiques, ou en tout cas variables 2 .

Il semble donc parfaitement concevable, surtout dans une période où les budgets de la santé sont passés à la loupe, que les «décideurs» demandent aux spécialistes de la prévention des méthodes moins onéreuses, dans la mesure du techniquement possible.

En deuxième lieu, en admettant que bien des maladies contemporaines prévalentes - maladies de la civilisation pour les uns (Schär [8]) ou maladies du libre choix pour d'autres (Lalonde [4]) - trouvent, sinon leur origine, du moins leur mécanisme dans le comportement des individus ou des groupes, on admet du même coup que leur prévention doit également passer par la sphère comportementale: un comportement nocif est donc à remplacer par un comportement bénéfique pour la santé. Or, qui dit modification du comportement pense immédiatement au modèle classique de Cartwright [1], lequel implique des changements sur trois plans:

- cognitif: ce que les gens savent ou comprennent,

- affectif: ce que les gens ont l'intention de faire,

- del'action: ce que les gens font effectivement.

En fait, c'est sur ce modèle que reposent depuis longtemps les principes d'action de l'éducation sanitaire (cf. plusieurs articles dans ce numéro et, en plus, celui de $M$. L. Collins [2]. Donc, notre éducation pour la santé représente bien la méthode par excellence d'approcher la lutte contre ces maladies.

En troisième lieu, l'apparition récente sur ce continent des mouvements de consommateurs («consumérisme») et du concept de participation aux décisions - assortis aux notions d'autogestion de la santé (OMS, 11), de "self care» [5] et même de "self-health» [7] - semble inévitablement impliquer la nécessité préalable pour les techniciens de la santé de transmettre au moins des connaissances aux populations ou groupes-cibles; d'autant plus que ceux-ci ne se limitent plus aux traditionnels décideurs ${ }^{3}$.

Dans cette perspective relativement nouvelle, il apparaît à l'évidence que l'éducation pour la santé doit pouvoir intervenir en priorité et en force dans cette transmission, à la condition de disposer, en temps et lieu, des ressources voulues en personnel et en techniques.

Mais, bien entendu, la réalité n'est ni aussi simple, ni aussi univoque. Voyons cela de plus près, brièvement.

A propos de la prévention secondaire, d'abord: il importe de rappeler que seul le dépistage peut intervenir comme mesure préventive utile dans le cas d'affections comme le cancer du col utérin; certes, fera-t-on remarquer, il faut que les femmes prennent l'habitude de s'y présenter, donc l'éducation intervient... En fait, l'obligation légale suffirait!

Concernant ensuite l'intervention dans le domaine du comportement, on doit, par souci d'équité, mentionner que plusieurs auteurs ont émis des doutes sur la légitimité du passage aisé du «vouloir faire» au «faire» (cf. p. ex. Kalb [3]).

Enfin, rien ne prouve que les partisans du «self care», de l'autogestion en matière de santé, etc., vont tous accueillir les messages de l'éducation pour la santé avec ferveur et reconnaissance, comme cela semble le cas en République populaire de Chine. On peut s'attendre au contraire, en Occident du moins, à des réactions vis-à-vis de ce que l'on pourrait voir désigner sous le terme de nouvelle forme d'impérialisme médical!

'Médecin. Professeur à la Faculté de Médecine, Directeur de LInstitut de Médecine sociale et préventive de l'Université de Genève. quai Ernest Ansermet 20, CH-1205 Genève

2Mentionnons ici deux points de vue de sources aussi différentes que possible: les conclusions prudentes d'une équipe fort connue dans le monde entier: celle de la Kayser Fondation [10]; et l'opinion nuancée du «patron» de la Sécurité sociale en France, le professeur Charles Sournia [9].

${ }^{3}$ Un intéressant exemple concernant les travailleurs de l'industrie ressort d'une communication italienne à la IXe conférence internationale d'éducation pour la santé [6]. 
Percevant aussi bien ces grandes tendances que ces réserves, la Rédaction a jugé opportun de reprendre ce vaste thème et de lui consacrer un numéro double ${ }^{4}$. Fallait-il se limiter aux principes, aux bases ou aborder les applications? Ici, les avis divergeaient. Finalement, le rédacteur de ce numéro a estimé judicieux d'ouvrir les colonnes de la Revue aux praticiens autant qu'aux théoriciens, dans l'idée que des expériences en cours, chez nos voisins comme dans notre pays, méritaient d'être mieux connues.

Une première partie met donc l'accent sur les principes, mais aussi sur les connaissances et attitudes des populations vis-à-vis de la santé, des messages et actions y relatifs et des acteurs en jeu. Dans cette optique, la contribution d'un sociologue, d'Houtaud, fournit des données factuelles qui apportent de l'eau au moulin de van Parijs, médecin belge spécialisé en éducation pour la santé, de H. R. M. Müller, jeune médecin bâlois, connu en Suisse pour ses travaux de sociologie médicale, et de $R$. Müller, collaborateur de l'ISPA 5 à Lausanne. Le lecteur verra ensuite à quel point les conceptions relatives à l'efficacité des moyens de communication de masse diffèrent chez les deux auteurs suivants, un sociologue de l'Université de Lausanne, Beaud, et un ingénieur français, Le Net, ex-délégué général du Comité français d'éducation pour la santé.

Dans la deuxième partie, on a tenu à demander à des médecins de situer les modalités si diverses de l'éducation pour la santé dans des contextes très différents: cadre d'un Centre de médecine préventive (Deschamps) et cadre scolaire (Ducommun et Hazeghi; Abelin et coll.); contexte de l'assurance-maladie (van Parijs), dans lequel la prévention secondaire est généralement davantage prise en considération.

Une troisième partie, consacrée spécifiquement à l'évaluation des résultats, comporte finalement quatre contributions: celle de Le Net (France), celle d'un groupe interdisciplinaire bâlois (Erne \& coll.), celle de Biener, médecin et éducateur (Zurich), toutes de la plus haute actualité, précédées d'un exposé introductif général d'un pionnier de l'éducation sanitaire en Suisse, notre collègue Schär. Deux autres devaient prendre place dans cette partie, mais leurs auteurs ont laissé entendre que cette partie de leur travail n'était pas encore assez avancée.

En fin de ce numéro, le lecteur trouvera de précieuses indications sur des institutions intemationales et sur une institution nationale (mais insuffisamment connue en Suisse romande!) ainsi qu'une liste de récentes publications de l'OMS (Bureau européen) dans ce domaine. Il appréciera à sa juste valeur la vivante relation de la IXe Conférence internationale de 1976 sortie de la plume alerte du Dr Jean Martin, un des rares représentants de notre pays à Ottawa!

Une moisson aussi riche et variée de contributions presque toutes originales fournissent toutes raisons de croire à l'avenir de l'éducation pour la santé, pour autant que cette discipline évolue dans le sens de la rigueur scientifique et du souci permanent de l'évaluation; cette moisson nous donne également bien des raisons d'espérer que, dans ce domaine aussi, notre pays participera davantage que jusqu'ici, aux initiatives et aux efforts des organismes internationaux concernés.

${ }^{4}$ Voir L,avertissement aux lecteurs, p. 209

Institut suisse pour la prophylaxie de l'alcoolisme

\section{Références bibliographiques}

[1] Cartwright, D., (1949) cité par M. L. Collins (1977).

[2] Collins, M. L., Behaviour modification techniques: their use in individual and community programmes. Internat. J. Health Educ., 20, 29-37, 1977.

[3] Kalb, M., The myth of alcoholism prevention Prev. Med. 4, 404-416, 1975.

[4] Lalonde, M., New perspectives on the health of Canadians: a working document, Ottawa, Ministry of Health and Welfare, Canada, 1974.

[5] Levin, L. S., Katz, A. H., et Holst, E., Self care: lay initiatives in health. New York, Prodist, 1976.

[6] Modolo, M. A., et Figà-Talamanca, I., Interaction between consumers and providers of health services: new roles and their implications. Internat. J. Health Educ. 20, 41-47, 1977.

[7] Ramakrishna, $V$., The real challenge: a self-health system with genuine commitment. Internat. J. Health Educ. 20, 19$28,1977$.

[8] Schär, M., Präventiv- und Sozialmedizin, Bern, H. Huber, 2. ed., 1973

[9] Sournia, Ch., Ces malades qu'on fabrique - la médecine gaspillée $(\mathrm{Ch}$. VI: la prévention médicale, mirages et réalités) Paris, le Seuil, 1977.

[10] Status Report on Multiphasic Health Testing. Prev. med. 2, 175-301, 1973.

[11] WHO, Report of a WHO Expert Committee on Planning and Evaluation of Health Education Services. Geneva, Techn. Rep. Series No. 409, 1969. 